\section{Zur Kenntnis des Verhaltens von Tetraphenyl- diphosphin gegenüber Carbonylverbindungen von Vanadin, Mangan und Kobalt}

\section{W. Hieber und R. Kummer}

Anorganisch-Chemisches Laboratorium der T.H. München

(Z. Naturforschg. 20 b, 271 [1965] ; eingegangen am 17. Dezember 1964)

Umsetzungen von Diphosphinen, $\mathrm{R}_{2} \mathrm{PPR}_{2}\left(\mathrm{R}=\mathrm{CH}_{3}\right.$, $\mathrm{C}_{2} \mathrm{H}_{5}$ und $\mathrm{C}_{6} \mathrm{H}_{5}$ ) und analogen Diarsinen mit Metallcarbonylen führen, wie Hayter et al. ${ }^{1}$ und Chatt et al. ${ }^{2}$ zeigten, je nach Versuchsbedingungen zu zweikernigen Carbonylen mit $\mu$-Diphosphino- bzw. $\mu$-Diarsinobrücken und $\mu$-Phosphido- bzw. $\mu$-Arsenidobrücken. Die Komplexe mit $\mu$-Diphosphinobrücken entstehen im allgemeinen primär unter milderen Bedingungen, wie neuerdings auch IssLeIB und $\mathrm{KeIL}^{3}$ zeigten. Unabhängig hiervon wurde von uns u. a. das Verhalten von Tetraphenyldiphosphin gegenüber Vanadincarbonyl, Vanadinnitrosylcarbonyl, Kobaltnitrosylcarbonyl und den Mangancarbonylhalogeniden geprüft.

Bei der Reaktion von Vanadincarbonyl mit Tetraphenyldiphosphin in Benzol entsteht unter CO-Entwicklung eine tief stahlblaue Verbindung, die sich mit dem bei der Reaktion von Vanadincarbonyl mit Diphenylphosphin schon früher ${ }^{4}$ festgestellten Produkt als identisch erwies, d.h. es handelt sich um Di- $\mu$-diphenylphosphido-bis (tetracarbonylvanadin), entsprechend der
Reaktion $\left(\mathrm{R}=\mathrm{C}_{6} \mathrm{H}_{5}\right)$

$2 \mathrm{~V}(\mathrm{CO})_{6}+\mathrm{R}_{2} \mathrm{PPR}_{2} \rightarrow(\mathrm{OC})_{4} \mathrm{~V}_{\mathrm{PR}_{2}}^{\prime} \mathrm{PR}_{2} \backslash \mathrm{V}(\mathrm{CO})_{4}+4 \mathrm{CO}$.

Die entsprechende Di- $\mu$-diphenylarsenido-Verbindung wurde inzwischen auf demselben Wege erhalten.

Auch Vanadinnitrosylcarbonyl reagiert in Cyclohexanlösung sehr leicht mit Tetraphenyldiphosphin, doch wurde nur der orangerote Zweikernkomplex mit dem Diphosphin,

$$
(\mathrm{OC})_{4}(\mathrm{NO}) \mathrm{V}\left(\mathrm{R}_{2} \mathrm{PPR}_{2}\right) \mathrm{V}(\mathrm{NO})(\mathrm{CO})_{4},
$$

erhalten, der infolge seiner Zersetzlichkeit keiner weiteren Umwandlung zugänglich ist.

Bei der Reaktion der Manganpentacarbonylhalogenide mit Tetraphenyldiphosphin unter milden $\mathrm{Be}$ dingungen (in Benzollösung bei $35^{\circ}$ bis $50^{\circ}$ ) erhält man nach Analyse und Mol.-Gew.-Bestimmung bemerkenswerterweise nur die einkernigen, gelben $(\mathrm{X}=\mathrm{Cl})$ bis roten $(\mathrm{X}=\mathrm{J})$ Verbindungen, entsprechend $(\mathrm{X}=$ $\mathrm{Cl}, \mathrm{Br}, \mathrm{J}$ )

$$
\mathrm{Mn}(\mathrm{CO})_{5}+\mathrm{R}_{2} \mathrm{PPR}_{2} \rightarrow \mathrm{X}(\mathrm{CO})_{4} \mathrm{Mn}\left(\mathrm{R}_{2} \mathrm{PPR}_{2}\right)+\mathrm{CO} .
$$

Läßt man die Komponenten im Molverhältnis 2:1 einige Stdn. in siedendem Benzol reagieren, so bilden sich die in gelben $(\mathrm{X}=\mathrm{Cl})$ bis braunen $(\mathrm{X}=\mathrm{J})$ Kristallen anfallenden zweikernigen Verbindungen mit $\mu$-Phosphidostruktur :

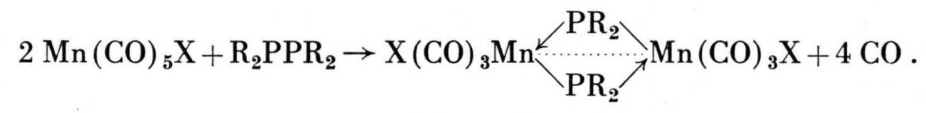

Kobaltnitrosylcarbonyl reagiert mit Tetraphenyldiphosphin zunächst unter Bildung eines roten Zweikernkomplexes mit Diphosphinbrücke, der sich erst bei mehrtägigem Erhitzen in $p$-Xylollösung in die braunschwarze, schwerlösliche Verbindung mit Phosphidobrücken umwandelt :

$(\mathrm{OC})_{2}(\mathrm{ON}) \mathrm{Co}\left(\mathrm{R}_{2} \mathrm{PPR}_{2}\right) \mathrm{Co}(\mathrm{NO})(\mathrm{CO})_{2} \rightarrow$

$$
\left[\mathrm{Co}(\mathrm{CO})(\mathrm{NO}) \mathrm{PR}_{2}\right]_{2}+2 \mathrm{CO} \text {. }
$$

1 R. G. Hayter, J. Amer. chem. Soc. 86, 823 [1964] ; Inorg. Chem. 3, 711 [1964] ; R. G. Hayter u. L. F. Williams, Inorg. Chem. 3, 717 [1964].

2 J. Chatt u. D. A. Thornton, J. chem. Soc. [London] 1964, 1005 ; J. Сhatt u. D. T. Thompson, J. chem. Soc. [London] 1964, 2713.
Nach dem IR-Spektrum - 1 CO-Valenzschwingung bei $2000 \mathrm{~cm}^{-1}$ und 1 NO-Valenzschwingung bei $1754 \mathrm{~cm}^{-1}$ - ist eindeutig auf trans-Konfiguration der CO- bzw. NO-Gruppen für letztere Verbindung (Punktgruppe $\mathrm{C}_{2 \mathrm{~h}}$ ) zu schließen.

Die Versuche werden in verschiedener Hinsicht fortgeführt.

3 K. Issleib u. M. Keil, Z. anorg. allg. Chem. 333, 10 [1964].

4 W. Hieber u. E. Winter, Chem. Ber. 97, 1037 [1964]. 